\title{
AN EFFICIENT LINEAR SOLVER FOR NONLINEAR PARAMETER IDENTIFICATION PROBLEMS*
}

\author{
YEE LO KEUNG ${ }^{\dagger}$ AND JUN ZOU
}

\begin{abstract}
In this paper, we study some efficient numerical methods for parameter identifications in elliptic systems. The proposed numerical methods are conducted iteratively and each iteration involves only solving positive definite linear algebraic systems, although the original inverse problems are ill-posed and highly nonlinear. The positive definite systems can be naturally preconditioned with their corresponding block diagonal matrices. Numerical experiments are presented to illustrate the efficiency of the proposed algorithms.
\end{abstract}

Key words. parameter identifications, elliptic problems, positive definite

AMS subject classifications. 35R30, 49K

PII. S1064827598346740

1. Introduction. The purpose of this paper is to investigate some numerical methods for efficiently identifying the unknown coefficient $q$ in the following elliptic problem:

$$
-\nabla \cdot(q \nabla u)=f \quad \text { in } \quad \Omega ; \quad u=0 \quad \text { on } \quad \Gamma \text {. }
$$

The identifying process is carried out in a way that the solution $u$ matches its observation data $z$ optimally either in the $L^{2}$-norm or in the $H^{1}$-norm. Here $\Omega$ can be any bounded domain in $R^{d}, d=1,2$, or 3 , with piecewise smooth boundary $\Gamma$ and $f \in H^{-1}(\Omega)$ is given. Practically, we are often asked to recover the parameter $q(x)$ using the observed data $z$ of the solution $u$. About the data $z$ we are interested in the following two cases:

(a) the measurement of the gradient of $u$ is available,

(b) the measurement of the solution $u$ itself is available.

For parameter identifications, Itô and Kunisch proposed a hybrid method in [11, $12,13]$ which combines the output least squares and the equation error formulation within the mathematical framework given by the augmented Lagrangian technique and incorporates a regularization term of the $H^{2}$-seminorm of the parameters to be recovered. Chen and Zou [5] and Keung and Zou [14] generalized the method to the case which allows the identifying coefficients to be discontinuous by using the regularization of bounded variations and they provided the rigorous theoretical justifications of the method and its finite element approximation. Independently, Chan and Tai $[3,4,18]$ considered also the regularization of bounded variations and did numerous experiments on the performance of the augmented Lagrangian method for identifying highly discontinuous parameters.

The primary approach we are interested in here is based on the following energy-

${ }^{*}$ Received by editors November 6, 1998; accepted for publication (in revised form) June 18, 2000; published electronically November $17,2000$.

http://www.siam.org/journals/sisc/22-5/34674.html

${ }^{\dagger}$ Pui Tak Canossian College, 200 Peel Rise, Aberdeen, Hong Kong.

${ }^{\ddagger}$ Department of Mathematics, The Chinese University of Hong Kong (zou@math.cuhk.edu.hk). Shatin, N.T., Hong Kong. The work of this author was partially supported by Hong Kong RGC grants CUHK 338/96E and CUHK 4004/98P and CUHK Direct Grant. 
norm least squares formulation of the aforementioned parameter identifying problem:

$$
\text { (P1) }\left\{\begin{array}{l}
\text { minimize } \quad J(q, v)=\frac{1}{2} \int_{\Omega} q|\nabla v-\nabla z|^{2} d x+\beta N(q) \\
\text { subject to } \quad(q, v) \in K \times V \text { and } \quad e(q, v)=0
\end{array}\right.
$$

in case (a) or on the following $L^{2}$-norm least squares formulation:

$$
\text { (P2) }\left\{\begin{array}{l}
\text { minimize } \quad J(q, v)=\frac{1}{2} \int_{\Omega}|v-z|^{2} d x+\beta N(q) \\
\text { subject to } \quad(q, v) \in K \times V \text { and } \quad e(q, v)=0
\end{array}\right.
$$

in case (b). Here $V=H_{0}^{1}(\Omega)$ and $K$ is a constrained set given by

$K=\left\{q \in L^{1}(\Omega) ; \quad N(q)<\infty\right.$ and $\alpha_{1} \leq q(x) \leq \alpha_{2} \quad$ almost everywhere (a.e.) in $\left.\Omega\right\}$

with $\alpha_{1}$ and $\alpha_{2}$ being two positive constants. $N(q)$ is a regularization term with a weighted coefficient $\beta>0 . e(\cdot, \cdot)$ is an operator from $K \times V$ into $V$ defined by the residual equation of (1.1) (in the weak sense):

$$
(\nabla e(q, v), \nabla \phi)=(q \nabla v, \nabla \phi)-(f, \phi) \quad \forall(q, v) \in K \times V, \quad \phi \in V,
$$

where $(\cdot, \cdot)$ denotes the duality pairing between $H^{-1}(\Omega)$ and $H_{0}^{1}(\Omega)$, which is the extension of the inner product in $L^{2}(\Omega)$. It is useful to remark that $e(q, v)$ is convex with respect to each variable.

The intention of this paper is to investigate some efficient and easy-to-implement method for the preceding parameter identifications. We know that the regularization of bounded variations, i.e., $N(q)=|q|_{B V(\Omega)}$, is very effective in recovering discontinuous parameters, but it also adds a big difficulty to the numerical resolution process. Namely, one has to solve a nearly singular and indefinite nonlinear minimization system of the form

$$
-\beta \nabla \cdot \frac{\nabla q}{\sqrt{|\nabla q|^{2}+\varepsilon}}+c(q)=g
$$

at each iteration, where $\varepsilon$ is the smoothing parameter introduced to smooth the BVnorm term in numerical implementations and $c(q)$ is a linear function of $q$, which causes the indefiniteness of the system; see $[3,4,5,14,18]$. It seems there are very few iterative methods which are known to be globally convergent for solving such a troublesome system. We refer to Chan and Mulet [2], Dobson and Vogel [8], and the references therein for some fixed point methods and their global convergence results when $c(q)$ is not too complicated.

In this paper, instead of the $B V$-norm regularization we are going to utilize the $H^{1}$ - or piecewise $H^{1}$-norm regularization. We will see that the $H^{1}$-norm regularization performs perfectly for identifying smooth coefficients as expected. In fact, our experience indicates that it can also give rise to satisfactory results in most discontinuous coefficient cases. But in the cases where the location of the discontinuities of the identifying coefficients is available, we can achieve much more accurate recoveries even for the coefficients with high discontinuities by using the piecewise $H^{1}$-norm regularization. The location of the discontinuities of parameters may be known in some applications or can be identified first by some existing simple algorithms. As we will show later on, with the $H^{1}$ - or piecewise $H^{1}$-norm regularization, each iteration of our algorithms involves only solving linear positive definite algebraic systems, which can be solved by the well-known GMRES iteration with guaranteed convergence [9]. Moreover, the positive definite systems may be preconditioned by their 
natural block diagonal matrices, which are very cheap and easy to implement. The numerical experiments will also show that these preconditioners are effective.

The problems (P1) and (P2) will be solved by the augmented Lagrangian method which enables us to relax the residual constraint $e(q, u)=0$ and enhance the convexity of the objective functional. For the purpose we introduce the augmented Lagrangian functional $\mathcal{L}_{r}: K \times V \times V \rightarrow R$ by

$$
\mathcal{L}_{r}(q, v ; \mu)=J(q, v)+(\nabla \mu, \nabla e(q, v))+\frac{r}{2}\|\nabla e(q, v)\|_{L^{2}(\Omega)}^{2},
$$

where $r \geq 0$ is some given constant.

Following the same arguments as we used in [5] we have the following.

THEOREM 1.1. $\left(q^{*}, v^{*}\right) \in K \times V$ is a solution of the minimization problem (P1) or (P2) if and only if there exists a $\lambda^{*} \in V$ such that $\left(q^{*}, v^{*}, \lambda^{*}\right) \in K \times V \times V$ is a saddle-point of the augmented Lagrangian $\mathcal{L}_{r}: K \times V \times V \rightarrow R$, namely,

$$
\mathcal{L}_{r}\left(q^{*}, v^{*} ; \mu\right) \leq \mathcal{L}_{r}\left(q^{*}, v^{*} ; \lambda^{*}\right) \leq \mathcal{L}_{r}\left(q, v ; \lambda^{*}\right) \quad \forall(q, v, \mu) \in K \times V \times V .
$$

Remark 1.1. We refer to [5] for the proof of similar results as stated in Theorem 1.1 for the TV-regularization, and $[12,13]$ for other quadratic regularizations.

2. Discretization and augmented Lagrangian algorithms. We now consider the finite element discretization of the augmented Lagrangian $\mathcal{L}_{r}$ and derive a discrete saddle-point problem.

Let $\Omega$ be a polyhedral domain in $R^{d}, d=1,2$, or 3 , and $\left\{\mathcal{T}^{h}\right\}_{h>0}$ be a family of regular triangulations (cf. Ciarlet [7]) of the domain $\Omega$ with simplicial elements. Denote by $V_{h}$ the standard piecewise linear finite element space over the triangulation $\mathcal{T}^{h}$ and

$$
\stackrel{\circ}{V}_{h}=V_{h} \cap H_{0}^{1}(\Omega), \quad K_{h}=K \cap V_{h} .
$$

The standard nodal basis functions of $\stackrel{\circ}{V}_{h}$ and $V_{h}$ are denoted as $\left\{\phi_{i}\right\}_{i=1}^{N_{0}}$ and $\left\{\phi_{i}\right\}_{i=1}^{N}$, respectively.

We define a discrete version of the operator $e(\cdot, \cdot): K \times V \rightarrow V$ as follows:

For any $\left(q_{h}, v_{h}\right) \in K_{h} \times \stackrel{\circ}{V}_{h}, e_{h}\left(q_{h}, v_{h}\right) \in \stackrel{\circ}{V}_{h}$ is the solution of the system

$$
\left(\nabla e_{h}\left(q_{h}, v_{h}\right), \nabla \phi\right)=\left(q_{h} \nabla v_{h}, \nabla \phi\right)-(f, \phi) \quad \forall \phi \in \stackrel{\circ}{V}_{h} .
$$

Then we introduce a discrete augmented Lagrangian $L_{r}$ from $K_{h} \times \stackrel{\circ}{V}_{h} \times \stackrel{\circ}{V}_{h}$ to $R$ :

$$
L_{r}\left(q_{h}, v_{h} ; \mu_{h}\right)=J_{h}\left(q_{h}, v_{h}\right)+\left(\nabla \mu_{h}, \nabla e_{h}\left(q_{h}, v_{h}\right)\right)+\frac{r}{2}\left\|\nabla e_{h}\left(q_{h}, v_{h}\right)\right\|_{L^{2}(\Omega)}^{2}
$$

with

$$
J_{h}\left(q_{h}, v_{h}\right)=\frac{1}{2} \int_{\Omega} q_{h}\left|\nabla v_{h}-\nabla z\right|^{2} d x+\beta \int_{\Omega}\left|\nabla q_{h}\right|^{2} d x
$$

in the case (a) and

$$
J_{h}\left(q_{h}, v_{h}\right)=\frac{1}{2} \int_{\Omega}\left|v_{h}-z\right|^{2} d x+\beta \int_{\Omega}\left|\nabla q_{h}\right|^{2} d x
$$

in the case (b). 
Applying the arguments as we used in [5], we obtain the following.

THEOREM 2.1. For any $r \geq 0$, there exists at least a saddle-point for the discrete augmented Lagrangian $L_{r}: K_{h} \times \stackrel{\circ}{V}_{h} \times \stackrel{\circ}{V}_{h} \rightarrow R$. Moreover, each saddle-point $\left(q_{h}^{*}, v_{h}^{*}, \lambda_{h}^{*}\right)$ of $L_{0}$ is also a saddle-point of $L_{r}$ for any $r>0$.

We apply the algorithm of the following Uzawa type to find the saddle-points of the discrete augmented Lagrangian $L_{r}$ defined in (2.2).

Uzawa Algorithm. Given $\lambda^{0} \in \stackrel{\circ}{V}_{h}$. Then for $n \geq 0$, determine the pair $\left\{q^{n}, u^{n}\right\} \in K_{h} \times \stackrel{\circ}{V}_{h}$ such that

$$
L_{r}\left(q^{n}, u^{n} ; \lambda^{n}\right)=\min \left\{L_{r}\left(p, v ; \lambda^{n}\right) \quad \forall(p, v) \in K_{h} \times \stackrel{\circ}{V}_{h}\right\}
$$

and then compute $\lambda^{n+1}$ by

$$
\lambda^{n+1}=\lambda^{n}+\rho_{n} e_{h}\left(q^{n}, u^{n}\right) .
$$

This Uzawa algorithm was proved in [5] to be convergent globally as long as $0<\rho_{n}<r$. Earlier proofs of such global convergence of the Uzawa algorithms with different quadratic regularizations can be found in [15] and the references therein. The major cost of the algorithm is solving (2.3), a coupled system with $q^{n}$ and $u^{n}$ as unknowns. In our implementations, we will use the following alternative iteration for solving (2.3).

Modified Uzawa Algorithm. Given $\lambda^{0} \in \stackrel{\circ}{V}_{h}$ and $q^{0} \in K_{h}$. Set $n=1$.

1. Set $k=1$ and $q^{n, 0}=q^{n-1}$.

2. Compute $u^{n, k} \in \stackrel{\circ}{V}_{h}$ by solving

$$
L_{r}\left(q^{n, k-1}, u^{n, k} ; \lambda^{n-1}\right)=\min _{v_{h} \in V_{h}^{0}} L_{r}\left(q^{n, k-1}, v_{h} ; \lambda^{n-1}\right),
$$

and then compute $q^{n, k} \in V_{h}$ by solving

$$
L_{r}\left(q^{n, k}, u^{n, k} ; \lambda^{n-1}\right)=\min _{p_{h} \in V_{h}} L_{r}\left(p_{h}, u^{n, k} ; \lambda^{n-1}\right) .
$$

Compute $q^{n, k}=\max \left\{\alpha_{1}, \min \left\{q^{n, k}, \alpha_{2}\right\}\right\}$.

If $\left\|q^{n, k}-q^{n, k-1}\right\| \leq$ tolerance, set $u^{n}=u^{n, k}$ and $q^{n}=q^{n, k}$, GOTO 3;

Otherwise set $k=k+1$, GOTO 2.

3. Compute $\lambda^{n}$ by

$$
\lambda^{n}=\lambda^{n-1}+\frac{3}{4} r e_{h}\left(q^{n}, u^{n}\right) .
$$

Set $n=n+1$, GOTO 1.

We will show in the next section that solving the minimization problems (2.5) and (2.6) is equivalent to solving two linear positive definite systems.

2.1. Positive definite systems: Energy-norm case. From the modified Uzawa algorithm we see that the major cost in each iteration of Step 2 is to solve the minimization problems (2.5) and (2.6). We next show that (2.5) and (2.6) are equivalent to two positive definite systems and thus can be solved using the GMRES iteration $[16,17]$, and more efficiently solved by the preconditioned GMRES 
method with their corresponding block diagonal symmetric positive definite matrices as preconditioners.

We first need to derive Gâteaux derivatives of the augmented Lagrangian functional $L_{r}(q, u ; \lambda)$. For the function $e_{h}\left(q_{h}, u_{h}\right)$, we can easily see its derivative with respect to $q_{h}$, denoted as $e_{h}^{\prime}\left(q_{h}, u_{h}\right) p_{h}$ at direction $p_{h} \in V_{h}$, is a finite element function in $\stackrel{\circ}{V}_{h}$ and solves the equation

$$
\left(\nabla e_{h}^{\prime}\left(q_{h}, u_{h}\right) p_{h}, \nabla \phi\right)=\left(p_{h} \nabla u_{h}, \nabla \phi\right) \quad \forall \phi \in \stackrel{\circ}{V}_{h},
$$

while its derivative with respect to $u_{h}$, denoted as $e_{h}^{\prime}\left(q_{h}, u_{h}\right) w_{h}$ at direction $w_{h} \in \stackrel{\circ}{V}_{h}$, is a finite element function in $\stackrel{\circ}{V}_{h}$ and solves the equation

$$
\left(\nabla e_{h}^{\prime}\left(q_{h}, u_{h}\right) w_{h}, \nabla \phi\right)=\left(q_{h} \nabla w_{h}, \nabla \phi\right) \quad \forall \phi \in \stackrel{\circ}{V}_{h} .
$$

Note from above that for the sake of notation, we distinguish between the directional derivatives of $e_{h}\left(q_{h}, u_{h}\right)$ with respect to $q_{h}$ and $u_{h}$ only by the direction notation, i.e., depending only on whether we use $p_{h}$ or $w_{h}$. Using these derivatives of $e_{h}\left(q_{h}, u_{h}\right)$, we can immediately obtain the Gâteaux derivatives of the augmented Lagrangian functional $L_{r}(q, u ; \lambda)$. Its derivative with respect to $u_{h}$ at direction $w_{h}$ is

$$
\begin{aligned}
& L_{r}^{\prime}\left(q_{h}, u_{h} ; \lambda_{h}\right) w_{h} \\
& =\left(q_{h}\left(\nabla u_{h}-\nabla z\right), \nabla w_{h}\right)+\left(\nabla \lambda_{h}, \nabla e_{h}^{\prime}\left(q_{h}, u_{h}\right) w_{h}\right) \\
& \quad+r\left(\nabla e_{h}\left(q_{h}, u_{h}\right), \nabla e_{h}^{\prime}\left(q_{h}, u_{h}\right) w_{h}\right) \\
& =\left(q_{h}\left(\nabla u_{h}-\nabla z\right), \nabla w_{h}\right)+\left(q_{h} \nabla \lambda_{h}, \nabla w_{h}\right)+r\left(q_{h} \nabla e_{h}\left(q_{h}, u_{h}\right), \nabla w_{h}\right),
\end{aligned}
$$

while its derivative with respect to $q_{h}$ at direction $p_{h}$ is

$$
\begin{aligned}
L_{r}^{\prime}\left(q_{h}, u_{h} ; \lambda_{h}\right) p_{h} & \\
=\frac{1}{2} \int_{\Omega} p_{h}\left|\nabla u_{h}-\nabla z\right|^{2} d x+\beta\left(\nabla q_{h},\right. & \left.\nabla p_{h}\right)+\left(\nabla \lambda_{h}, \nabla e_{h}^{\prime}\left(q_{h}, u_{h}\right) p_{h}\right) \\
+ & r\left(\nabla e_{h}\left(q_{h}, u_{h}\right), \nabla e_{h}^{\prime}\left(q_{h}, u_{h}\right) p_{h}\right) \\
=\frac{1}{2} \int_{\Omega} p_{h}\left|\nabla u_{h}-\nabla z\right|^{2} d x+\beta\left(\nabla q_{h},\right. & \left.\nabla p_{h}\right)+\left(p_{h} \nabla u_{h}, \nabla \lambda_{h}\right) \\
+ & r\left(p_{h} \nabla u_{h}, \nabla e_{h}\left(q_{h}, u_{h}\right)\right) .
\end{aligned}
$$

Now combining the formula $(2.10)$ and the definition of $e_{h}(\cdot, \cdot)$, we can find the solution $u^{n, k}$ in (2.5), together with $e_{h}\left(q^{n, k-1}, u^{n, k}\right)$ as follows:

Find $\left(u^{n, k}, e_{h}\left(q^{n, k-1}, u^{n, k}\right)\right) \equiv\left(u_{h}, e_{h}\right) \in \stackrel{\circ}{V}_{h} \times \stackrel{\circ}{V}_{h}$ such that

$$
\begin{aligned}
\left(q^{n, k-1} \nabla u_{h}, \nabla w_{h}\right)+r\left(q^{n, k-1} \nabla e_{h}, \nabla w_{h}\right) & =\left(q^{n, k-1} \nabla\left(z-\lambda^{n-1}\right), \nabla w_{h}\right), \\
\left(\nabla e_{h}, \nabla \phi_{h}\right)-\left(q^{n, k-1} \nabla u_{h}, \nabla \phi_{h}\right) & =-\left(f, \phi_{h}\right)
\end{aligned}
$$

$\forall w_{h} \in \stackrel{\circ}{V}_{h}$ and $\phi_{h} \in \stackrel{\circ}{V}_{h}$. Similarly, the solution $q^{n, k}$ in (2.6) can be solved together with $e_{h}\left(q^{n, k}, u^{n, k}\right)$ :

Find $\left(q^{n, k}, e_{h}\left(q^{n, k}, u^{n, k}\right)\right) \equiv\left(q_{h}, e_{h}\right) \in V_{h} \times \stackrel{\circ}{V}_{h}$ such that

$$
\begin{aligned}
\beta\left(\nabla q_{h}, \nabla p_{h}\right)+r\left(\nabla e_{h}, p_{h} \nabla u^{n, k}\right) & =-g_{k}\left(p_{h}\right), \\
\left(\nabla e_{h}, \nabla \phi_{h}\right)-\left(q_{h} \nabla u^{n, k}, \nabla \phi_{h}\right) & =-\left(f, \phi_{h}\right)
\end{aligned}
$$


$\forall p_{h} \in V_{h}$ and $\phi_{h} \in \stackrel{\circ}{V}_{h}$. Here $g_{k}\left(p_{h}\right)$ is given by

$$
g_{k}\left(p_{h}\right)=\frac{1}{2} \int_{\Omega} p_{h}\left|\nabla\left(u^{n, k}-z\right)\right|^{2} d x+\left(p_{h} \nabla u^{n, k}, \nabla \lambda^{n-1}\right) .
$$

Next we show that the linear systems (2.12)-(2.13) and (2.14)-(2.15) are both positive definite. To see this, we introduce

$$
\mathbf{A}=\left(a_{i j}\right), \quad \mathbf{B}=\left(b_{i j}\right), \quad \mathbf{\Lambda}=\left(\Lambda_{j}\right), \quad \mathbf{F}=\left(f_{j}\right)
$$

with

$$
a_{i j}=\left(q^{n, k-1} \nabla \phi_{i}, \nabla \phi_{j}\right), \quad b_{i j}=\left(\nabla \phi_{i}, \nabla \phi_{j}\right)
$$

and

$$
\Lambda_{j}=\left(q^{n, k-1} \nabla\left(z-\lambda^{n-1}\right), \nabla \phi_{j}\right), \quad f_{j}=\left(f, \phi_{j}\right)
$$

for $i, j=1,2, \ldots, N_{0}$. Then $(2.12)-(2.13)$ can be written as follows:

$$
\mathbf{A u}+r \mathbf{A e}=\mathbf{\Lambda}, \quad \mathbf{B e}-\mathbf{A} \mathbf{u}=-\mathbf{F},
$$

or equivalently

$$
(\mathbf{B}+r \mathbf{A}) \mathbf{e}=\mathbf{\Lambda}-\mathbf{F}, \quad \mathbf{A} \mathbf{u}=\mathbf{B} \mathbf{e}+\mathbf{F},
$$

where $\mathbf{u}$ and $\mathbf{e}$ are the coefficient vectors of $u_{h}$ and $e_{h}$ in terms of the basis $\left\{\phi_{i}\right\}_{i=1}^{N_{0}}$, respectively. These two equations are both symmetric positive definite and can be solved by the conjugate gradient methods, or more efficiently by PCG method with the standard domain decomposition type preconditioners [1].

In an analogous manner, we introduce the following notation for the system $(2.14)-(2.15)$

$$
\mathbf{Q}=\left(q_{i j}\right), \quad \mathbf{N}=\left(n_{i j}\right), \quad \mathbf{G}=\left(g_{k}\left(\phi_{j}\right)\right)
$$

with

$$
q_{i j}=\left(\nabla \varphi_{i}, \nabla \varphi_{j}\right), \quad n_{i j}=\left(\varphi_{i} \nabla u^{n, k}, \nabla \phi_{j}\right)
$$

for $i, j=1,2, \ldots, N$. Then $(2.14)-(2.15)$ can be written as follows:

$$
\beta \mathbf{Q} \mathbf{q}+r \mathbf{N} \mathbf{e}=-\mathbf{G}, \quad \mathbf{B} \mathbf{e}-\mathbf{N}^{\top} \mathbf{q}=-\mathbf{F} .
$$

By eliminating e, we can easily show that the system has a unique solution pair (q,e). For implementation, we write the system as

$$
\left(\begin{array}{cc}
r \mathbf{B} & -r \mathbf{N}^{\top} \\
r \mathbf{N} & \beta \mathbf{Q}
\end{array}\right)\left(\begin{array}{l}
\mathbf{e} \\
\mathbf{q}
\end{array}\right)=\left(\begin{array}{c}
-r \mathbf{F} \\
-\mathbf{G}
\end{array}\right)
$$

If we let $\mathbf{A}_{2}$ be the coefficient matrix of the above system, and $\mathbf{D}_{2}$ be the block diagonal matrix of $\mathbf{A}_{2}$, then we can easily verify that

$$
\left(\mathbf{e}^{\top}, \mathbf{q}^{\top}\right) \mathbf{A}_{2}\left(\begin{array}{l}
\mathbf{e} \\
\mathbf{q}
\end{array}\right)=r \mathbf{e}^{\top} \mathbf{B} \mathbf{e}+\beta \mathbf{q}^{\top} \mathbf{Q} \mathbf{q}=\left(\mathbf{e}^{\top}, \mathbf{q}^{\top}\right) \mathbf{D}_{2}\left(\begin{array}{l}
\mathbf{e} \\
\mathbf{q}
\end{array}\right)>0
$$


for any nonzero pair $(\mathbf{q}, \mathbf{e})$. Therefore $\mathbf{A}_{2}$ is positive definite, and so (2.17) can be solved using the GMRES iterative method, or more efficiently solved using the preconditioned GMRES method. A natural choice of the preconditioner matrix for $\mathbf{A}_{2}$ is $\mathbf{D}_{2}^{-1}$, with

$$
\mathbf{D}_{2}=\left(\begin{array}{cc}
r \mathbf{B} & \\
& \beta \mathbf{Q}
\end{array}\right)
$$

which is independent of the number of iterations and so is very cheap and easy to implement.

2.2. Positive definite systems: $\boldsymbol{L}^{\mathbf{2}}$-norm case. In the $L^{2}$-norm case, we can similarly obtain the Gâteaux derivative of the augmented Lagrangian functional with respect to $u_{h}$ and $q_{h}$ as in section 2.1. The derivative with respect to $u_{h}$ at direction $w_{h}$ is

$$
L_{r}^{\prime}\left(q_{h}, u_{h} ; \lambda_{h}\right) w_{h}=\left(u_{h}-z, w_{h}\right)+\left(q_{h} \nabla \lambda_{h}, \nabla w_{h}\right)+r\left(q_{h} \nabla e_{h}\left(q_{h}, u_{h}\right), \nabla w_{h}\right),
$$

while its derivative with respect to $q_{h}$ at direction $p_{h}$ is

$$
L_{r}^{\prime}\left(q_{h}, u_{h} ; \lambda_{h}\right) p_{h}=\beta\left(\nabla q_{h}, \nabla p_{h}\right)+\left(p_{h} \nabla u_{h}, \nabla \lambda_{h}\right)+r\left(p_{h} \nabla u_{h}, \nabla e_{h}\left(q_{h}, u_{h}\right)\right)
$$

Now combining the formula (2.19) and the definition of $e_{h}(\cdot, \cdot)$, we can find the solution $u^{n, k}$ in (2.5), together with $e_{h}\left(q^{n, k-1}, u^{n, k}\right)$, as follows:

Find $\left(u^{n, k}, e_{h}\left(q^{n, k-1}, u^{n, k}\right)\right) \equiv\left(u_{h}, e_{h}\right) \in \stackrel{\circ}{V}_{h} \times \stackrel{\circ}{V}_{h}$ such that

$$
\begin{aligned}
\left(u_{h}, w_{h}\right)+r\left(q^{n, k-1} \nabla e_{h}, \nabla w_{h}\right) & =\left(z, w_{h}\right)-\left(q^{n, k-1} \nabla \lambda^{n-1}, \nabla w_{h}\right), \\
\left(\nabla e_{h}, \nabla \phi_{h}\right)-\left(q^{n, k-1} \nabla u_{h}, \nabla \phi_{h}\right) & =-\left(f, \phi_{h}\right)
\end{aligned}
$$

$\forall w_{h} \in \stackrel{\circ}{V}_{h}$ and $\phi_{h} \in \stackrel{\circ}{V}_{h}$.

We next show that $(2.21)-(2.22)$ is a positive definite system. To see this, we introduce

$$
\mathbf{A}=\left(a_{i j}\right), \quad \mathbf{B}=\left(b_{i j}\right), \quad \mathbf{M}=\left(m_{i j}\right), \quad \mathbf{H}=\left(h_{j}\right), \quad \mathbf{F}=\left(f_{j}\right)
$$

with

$$
a_{i j}=\left(q^{n, k-1} \nabla \phi_{i}, \nabla \phi_{j}\right), \quad b_{i j}=\left(\nabla \phi_{i}, \nabla \phi_{j}\right), \quad m_{i j}=\left(\phi_{i}, \phi_{j}\right)
$$

and

$$
h_{j}=\left(z, \phi_{j}\right)-\left(q^{n, k-1} \nabla \lambda^{n-1}, \nabla \phi_{j}\right), \quad f_{j}=\left(f, \phi_{j}\right),
$$

for $i, j=1,2, \ldots, N_{0}$. Then $(2.21)-(2.22)$ can be written as follows:

$$
\mathbf{M u}+r \mathbf{A e}=\mathbf{H}, \quad \mathbf{B e}-\mathbf{A u}=-\mathbf{F},
$$

or equivalently,

$$
\left(\begin{array}{cc}
r \mathbf{B} & -r \mathbf{A} \\
r \mathbf{A} & \mathbf{M}
\end{array}\right)\left(\begin{array}{l}
\mathbf{e} \\
\mathbf{u}
\end{array}\right)=\left(\begin{array}{r}
-r \mathbf{F} \\
\mathbf{H}
\end{array}\right)
$$


Let $\mathbf{A}_{3}$ be the coefficient matrix of the above system and $\mathbf{D}_{3}$ be the block diagonal matrix of $\mathbf{A}_{3}$; then we can see that $\mathbf{A}_{3}$ is positive definite as

$$
\left(\mathbf{e}^{\top}, \mathbf{u}^{\top}\right) \mathbf{A}_{3}\left(\begin{array}{l}
\mathbf{e} \\
\mathbf{u}
\end{array}\right)=r \mathbf{e}^{\top} \mathbf{B} \mathbf{e}+\mathbf{u}^{\top} \mathbf{M} \mathbf{u}=\left(\mathbf{e}^{\top}, \mathbf{u}^{\top}\right) \mathbf{D}_{3}\left(\begin{array}{l}
\mathbf{e} \\
\mathbf{u}
\end{array}\right)>0
$$

for any nonzero pair (q,e). Thus the system can be solved by the GMRES method. Another way to solve the system is to first eliminate $\mathbf{u}$ and solve the symmetric positive definite system $\left(\mathbf{B}+r \mathbf{A} \mathbf{M}^{-1} \mathbf{A}\right) \mathbf{e}=-\mathbf{F}+\mathbf{A} \mathbf{M}^{-1} \mathbf{H}$ for $\mathbf{e}$ and then substitute e back to the system. Note that if we use the lumped mass scheme to approximate the mass matrix $\mathbf{M}$, then $\mathbf{M}$ is a diagonal matrix. This simplification keeps the same accuracy of approximation as we calculate $\mathbf{M}$ exactly (cf. Hoffmann-Zou [10]).

On the other hand, combining the formula $(2.20)$ and the definition of $e_{h}(\cdot, \cdot)$, we know that the solution $q^{n, k}$ in (2.6) satisfies the same equation as (2.17) but with $g_{k}\left(\phi_{j}\right)$ replaced by

$$
\int_{\Omega} \varphi_{i} \nabla u^{n, k} \cdot \nabla \lambda^{n-1} d x
$$

3. Regularization with piecewise $\boldsymbol{H}^{\mathbf{1}}$ seminorms. In some applications, the location of the discontinuities of the identifying parameters may be available, either achieved technically or obtained by some simple numerical methods. In this case, we propose to use the piecewise $H^{1}$-seminorm regularization to obtain a more accurate recovery for the highly discontinuous parameters.

For simplicity, let us assume that the coefficient $q(x)$ has jumps only across the interface $\Gamma$ between two subdomains $\Omega_{1}$ and $\Omega_{2}$ of $\Omega$, namely,

$$
q(x)= \begin{cases}q_{1}(x), & x \in \Omega_{1}, \\ q_{2}(x), & x \in \Omega_{2}\end{cases}
$$

with $\bar{\Omega}=\bar{\Omega}_{1} \cup \bar{\Omega}_{2}$. The corresponding residual equation (1.2) becomes

$$
(\nabla e(q, v), \nabla \phi)=(q \nabla v, \nabla \phi)-\left\{\int_{\Gamma}\left[q \frac{\partial u}{\partial \mathbf{n}}\right] \phi+(f, \phi)\right\} \quad \forall(q, v) \in K \times V, \quad \phi \in V .
$$

Here $\left[q \frac{\partial u}{\partial \mathbf{n}}\right]$ is the jump of the flux $q \frac{\partial u}{\partial \mathbf{n}}$ across the interface $\Gamma$, which is given and in fact vanishes in many real applications. We assume that $\Gamma$ is a piecewise line segment $(d=$ 2 ) or a polygon $(d=3)$. In other cases the finite element discretization needs to be taken care of very technically (cf. Chen-Zou [6]). Let $\mathcal{T}^{h}$ be a triangulation of $\Omega$ with all the finite elements aligning with the interface $\Gamma$ and $\tilde{V}_{h}$ be the standard piecewise linear finite element space, respectively, in $\Omega_{1}$ and $\Omega_{2}$, namely, with piecewise linear functions which are continuous both in $\Omega_{1}$ and $\Omega_{2}$ but probably having jumps across $\Gamma$. We define the discrete constrained set to be $\tilde{K}_{h}=K \cap \tilde{V}_{h}$.

Now we can reformulate the augmented Lagrangian functional $\mathcal{L}_{r}$ in (1.3) by replacing the regularization term $N(q)$ there by

$$
\tilde{N}(q)=\beta \int_{\Omega_{1}}\left|\nabla q_{1}\right|^{2} d x+\beta \int_{\Omega_{2}}\left|\nabla q_{2}\right|^{2} d x .
$$

And in this case the discrete constrained set is defined to be $\tilde{K}_{h}=K \cap \tilde{V}_{h}$, and the discrete augmented Lagrangian functional $L_{r}$ in (2.2) is now defined on $\tilde{K}_{h} \times \stackrel{\circ}{V}_{h} \times \stackrel{\circ}{V}_{h}$ 
and has the same form as (2.2) but with the regularization terms in $J_{h}\left(q_{h}, v_{h}\right)$ replaced by $\tilde{N}\left(q_{h}\right)$.

All the derivations in sections 2.1-2.2 can be carried over with only minor notational changes. The resulting linear algebraic systems from (2.5) and (2.6) are still all positive definite as we showed in sections $2.1-2.2$.

4. Numerical experiments. We now show some numerical experiments on the proposed method for parameter identifications. We apply the modified Uzawa algorithm for the identification of the coefficients in the following test problems:

$$
\begin{gathered}
-\frac{d}{d x}\left(q(x) \frac{d}{d x} u(x)\right)=f(x), \quad x \in(0,1), \\
u(0)=0, \quad u(1)=0
\end{gathered}
$$

and

$$
\begin{aligned}
-\nabla \cdot(q(x, y) \nabla u)+c(x, y) u & =f(x, y), & & (x, y) \in \Omega, \\
u(x, y) & =0, & & (x, y) \in \partial \Omega,
\end{aligned}
$$

where $\Omega=(0,1) \times(0,1)$. In our implementations, the interval $(0,1)$ is divided into $N$ uniformly distributed subintervals of length $h=1 / N$, the domain $(0,1) \times(0,1)$ is triangulated uniformly into triangular elements with horizontal and vertical edges of length $h=1 / N$.

Most parameters required in the algorithm are attached in each figure. The error $\mathcal{E}$ shown is the relative $L^{2}$-norm error between the exact parameter $q(x)$ to be identified and the numerically identified parameter $q_{h}$. The numerically recovered $q_{h}$ shown throughout this section are the result obtained at the 5 th iteration, i.e., $n=5$, in the modified Uzawa algorithm. The augmented Lagrangian coefficient $r$ and the initial guess of the Lagrangian multiplier $\lambda^{0}$ are always set to be 1 and 0 , and the finite element mesh size $h$ to be $1 / 80$ unless otherwise specified. The lower and upper bounds $\alpha_{1}$ and $\alpha_{2}$ in the constrained set $K$ are taken to be 0.5 and 20.0, respectively. The tolerance is taken to be $10^{-6}$, i.e., $\left\|q^{n, k}-q^{n, k-1}\right\|_{\infty} \leq 10^{-6}$.

To generate the noisy observation data, we will always take the following form:

$$
\nabla z^{\delta}(x)=\nabla z+\delta \operatorname{rand}(x)
$$

in case (a) or

$$
z^{\delta}(x)=z+\delta \operatorname{rand}(x)
$$

in case (b), where $\operatorname{rand}(x)$ is a uniformly distributed random (vector-valued in (a)) function in $[-1,1], \delta$ is the noise level parameter. Then in our implementations, we replace all previously appeared $\nabla z$ and $z$ by $\nabla z^{\delta}$ and $z^{\delta}$, respectively.

4.1. Energy-norm case. We first show some results for the energy-norm case. Example 1. We take the observed data $z$ as

with the coefficient $q(x)$ as

$$
z(x)=u(q)(x)=\sin (2 \pi x)
$$

$$
q(x)=3+2 x^{2}-2 \sin (2 \pi x) .
$$

Figures 1 and 2 show the exact solution $q(x)$ (the solid line in Figure 1 but dashed line in Figure 2) and the numerically identified solution $q_{h}(x)$ (the "o" line in Figure 


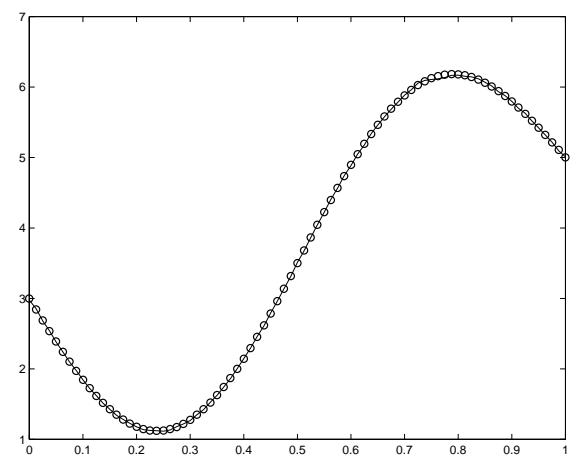

FIG. $1: q_{h}^{0}=5.0, \beta=10^{-5}, \delta=1 \%, \mathcal{E}=0.0025$.

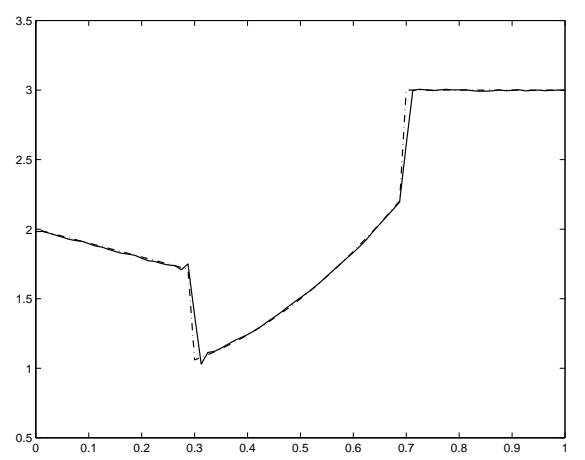

FIG. $3: q_{h}^{0}=5.0, \beta=5 \times 10^{-5}, \delta=1 \%, \mathcal{E}=0.026$.

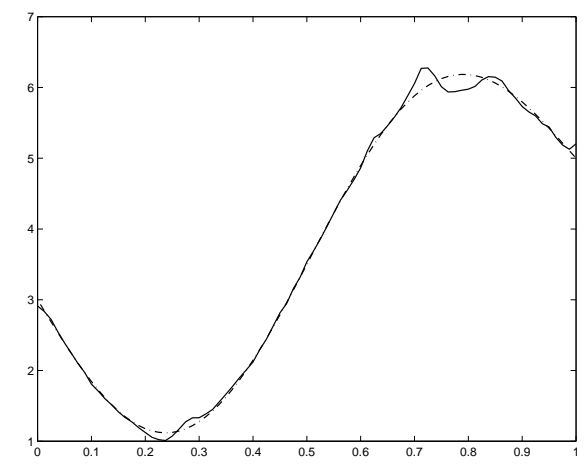

FIG. $2: q_{h}^{0}=5.0, \beta=10^{-5}, \delta=10 \%, \mathcal{E}=0.021$.

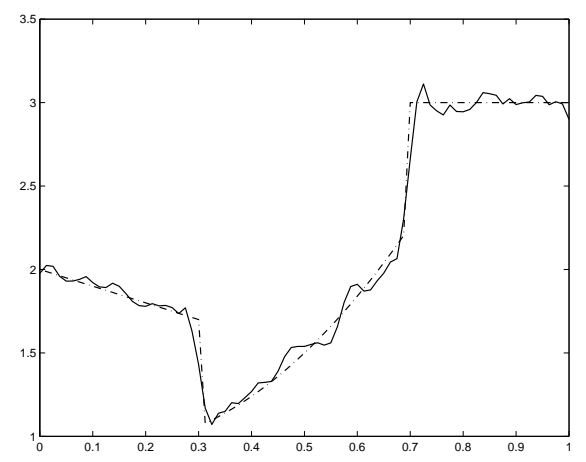

FIG. $4: q_{h}^{0}=5.0, \beta=10^{-5}, \delta=10 \%, \mathcal{E}=0.031$.

1 but solid line in Figure 2) with the noise level $\delta=1 \%$ and $\delta=10 \%$, respectively. Note that the initial guess $q_{h}^{0}=5.0$ is not a good initial guess at all, but the numerical method converges very stably and fast. Figures 1 and 2 are the results obtained at the 5th iteration $(n=5)$. As the numerical algorithm is a globally convergent algorithm, one can take the initial $q_{h}^{0}$ much worse than $q_{h}^{0}=5.0$, say, $q_{h}^{0}=50.0$, and still obtain the same accurate recovery. This is true for all examples shown in this section.

Example 2. We take the observed data $z$ as

$$
z(x)=u(q)(x)=\sin (\pi x)
$$

with the discontinuous coefficient $q(x)$ as

$$
q(x)= \begin{cases}2-x, & x \in[0,0.3] \\ 1-x+4 x^{2}, & x \in(0.3,0.7), \\ 3, & x \in[0.7,1] .\end{cases}
$$

Figures 3 and 4 show the exact solution $q(x)$ (the dashed line) and the numerically identified solution $q_{h}(x)$ (the solid line) with the noise level $\delta=1 \%$ and $10 \%$, respectively.

Example 3. We take the observed data $z$ as

$$
z(x, y)=u(q)(x, y)=\sin (\pi x) \sin (\pi y)
$$

and the coefficient $q(x, y)$ as

$$
q(x, y)=1+6 x^{2} y(1-y) .
$$




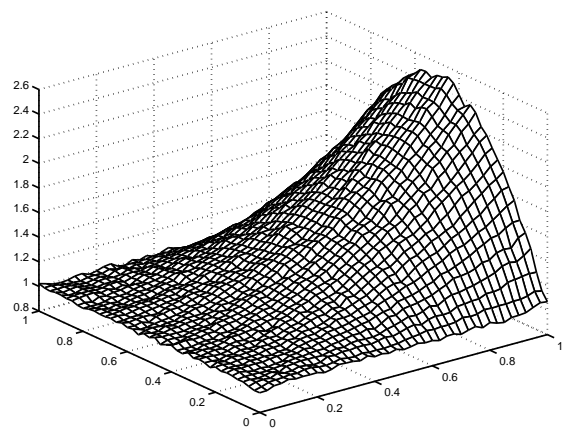

FIG. 5: $q_{h}^{0}=5.0, \beta=10^{-5}, \delta=1 \%, \mathcal{E}=0.025$.

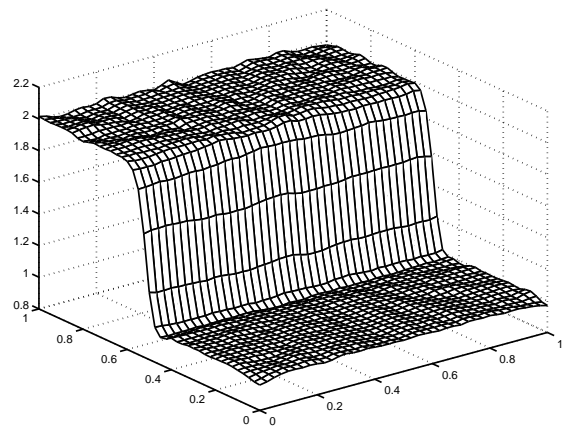

FIG. $7: q_{h}^{0}=5.0, \beta=10^{-5}, \delta=1 \%, \mathcal{E}=0.049$.

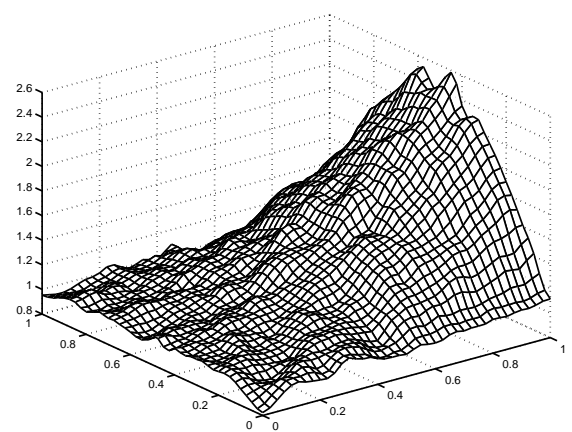

FIG. $6: q_{h}^{0}=5.0, \beta=10^{-4}, \delta=10 \%, \mathcal{E}=0.079$.

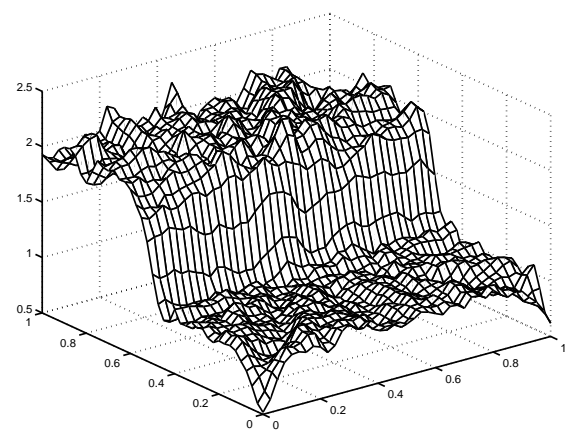

FIG. 8: $q_{h}^{0}=5.0, \beta=10^{-5}, \delta=10 \%, \mathcal{E}=0.069$.

Figures 5-6 show the numerically identified solution $q_{h}(x, y)$ with $h=1 / 40$ and the noise level $\delta=1 \%$ and $\delta=10 \%$, respectively.

Example 4. We take the observed data $z$ as

$$
z(x)=u(q)(x, y)=\sin (\pi x) \sin (\pi y)
$$

with a discontinuous coefficient $q(x, y)$ as

$$
\begin{cases}q(x, y)=1, & y \in[0,0.5] \\ q(x, y)=2, & y \in(0.5,1] .\end{cases}
$$

Figures 7-8 show the numerically identified solution $q_{h}(x, y)$ with $h=1 / 40$, and the noise level $\delta=1 \%$ and $\delta=10 \%$, respectively.

4.2. $L^{2}$-norm case. In this subsection, we show some numerical experiments on the proposed method with the $L^{2}$-norm formulation for the identification of the coefficient $q(x)$ in the test problems (4.1)-(4.2) and (4.3)-(4.4). The $L^{2}$-norm formulation assumes that the observation data is available via the pointwise function values, not the gradient values as in the energy-norm case. Note that the behavior of the gradient of a function is more essential for knowing the changes of a function than the function values, so in general we cannot expect the same nice performance in the current case as in the energy-norm case.

Example 5. The observed data $z$ and the coefficient $q(x)$ are the same as in Example 1. Figure 9 shows the exact solution $q(x)$ (the dashed line) and the numerically identified solution $q_{h}(x)$ (the solid line) with the noise level $\delta=1 \%$. 


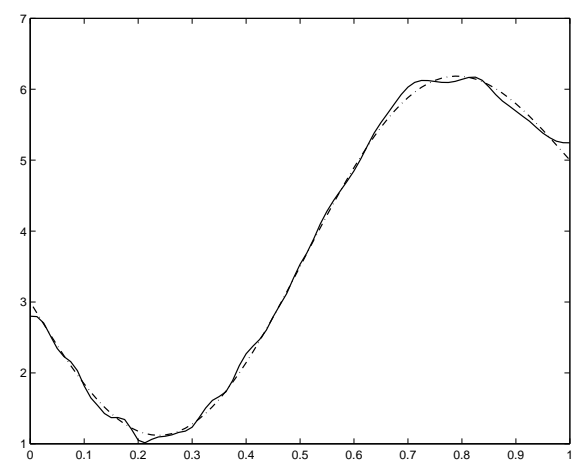

FIG. 9: $q_{h}^{0}=3.0, \beta=10^{-7}, \delta=1 \%, \mathcal{E}=0.018$.

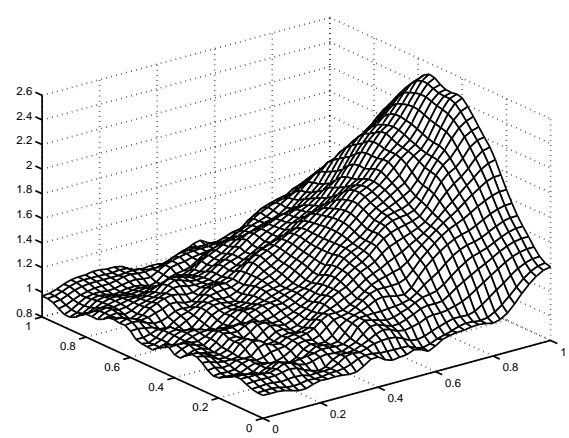

FIG. $10: q_{h}^{0}=5.0, \beta=10^{-6}, \delta=1 \%, \mathcal{E}=0.031$.

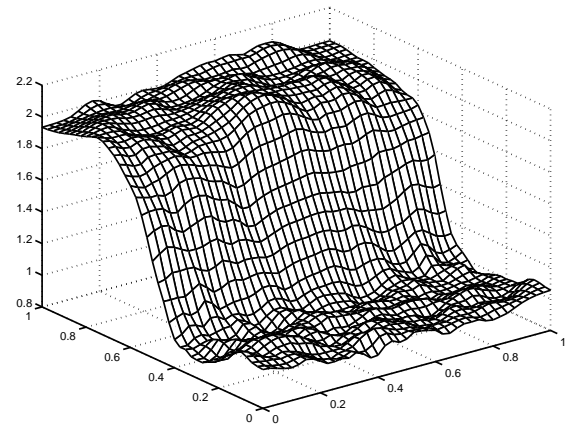

FIG. 11: $q_{h}^{0}=5.0, \beta=10^{-6}, \delta=1 \%, \mathcal{E}=0.091$.

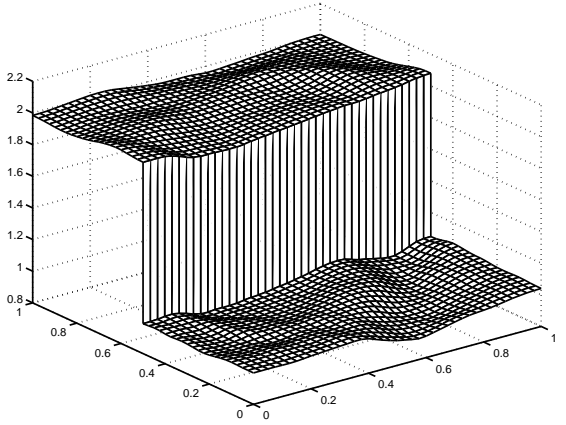

FIG. 12: $q_{h}^{0}=5.0, \gamma=10^{-4}, \delta=10 \%, \mathcal{E}=0.018$.

Example 6. The observed data $z$ and the discontinuous coefficient $q(x)$ are taken to be the same as in Example 3. Figure 10 shows the numerical solution $q_{h}(x, y)$ with $h=1 / 40$ and the noise level $\delta=1 \%$.

Example 7. The observed data $z$ and the discontinuous coefficient $q(x)$ are taken to be the same as in Example 4. Figure 11 shows the numerically identified solution $q_{h}(x, y)$ with $h=1 / 40$ and the noise level $\delta=1 \%$; the recovery seems acceptable but not very satisfactory.

If the location of the discontinuity of the parameter $q(x)$ is available, then we can achieve much better identification by using the piecewise $H^{1}$-seminorm regularization (see section 3). Figure 12 is the numerically identified results using piecewise $H^{1}$ seminorm regularization with the noise level $\delta=10 \%$ and $h=1 / 40$. Compared with 
Figure 11, where the noise level is only $\delta=1 \%$, we can see that the numerical results using piecewise $H^{1}$-seminorm regularization are much more satisfactory.

4.3. GMRES iteration. As stated in the modified Uzawa algorithm, we have to eventually solve two minimization problems (2.5) and (2.6) at each iteration of step 2. But we have shown in sections 2.1-2.2 that these two problems are both equivalent to solving two positive definite systems. In this section we take the energy-norm formulation as an example and present some detailed numerical performance of the Uzawa algorithm using the GMRES method to solve the nonsymmetric but positive definite system (2.17). From the GMRES theory we know this system can be solved by GMRES with guaranteed convergence. However, when discretization mesh size is very fine, (2.17) is large and still expensive to solve, especially in two and three dimensions. So good preconditioners are needed. Fortunately we have a cheap and natural preconditioner available, i.e., $M^{-1}$. Here $M$ is the block diagonal coefficient matrix

$$
M=\left(\begin{array}{cc}
r B & 0 \\
0 & \beta Q
\end{array}\right)
$$

of (2.17). We are going to demonstrate some numerical experiments and compare the results using GMRES with or without preconditioning.

In our implementation, the stopping criterion of all GMRES or preconditioned GMRES iteration for a system $A x=b$ is taken to be $10^{-6}$, i.e.,

$$
\frac{\|b-A x\|}{\|b\|}<10^{-6} \text {. }
$$

The system $M x=c$ involved in the preconditioned GMRES is always solved by the backward and forward substitutions using the LU factorization of $M$, i.e., $M=L U$. Note that the two diagonal block matrices $B$ and $Q$ of the preconditioner $M$ are both symmetric and positive definite, in fact they are the stiffness matrices arising from the finite element discretization of the simple Laplacian operator, so the preconditioner $M$ is unchanged in the whole outer (Uzawa iteration) and inner (GMRES) iterations. Thus the LU factorization of $M$ needs to be done only once for all, namely, it can be done before we start the Uzawa algorithm.

We remark that the matrix $Q$ used in the preconditioner $M$ is a singular matrix, so the upper triangular matrix $U$ of the $\mathrm{LU}$ factorization may have zeros on its diagonal. In this case, we add $10^{-10}$ (for $d=1$ ) and $10^{-6}$ (for $d=2$ ) to those diagonal entries with magnitudes less than $10^{-10}$.

Another way to avoid the singularity of the matrix $Q$ is to replace the seminorm $\int_{\Omega}|\nabla q|^{2} d x$ by the full-norm $\int_{\Omega}\left(|\nabla q|^{2}+q^{2}\right) d x$ as the regularization term. In this case,

$$
Q=\left(q_{i j}\right), \quad q_{i j}=\left(\nabla \varphi_{i}, \nabla \varphi_{j}\right)+\left(\varphi_{i}, \varphi_{j}\right)
$$

and the preconditioner $\tilde{M}$ formed as in (4.5) is always nonsingular.

In the following examples, we will demonstrate the effectiveness of the preconditioner $M$ and the effect of seminorm and full-norm regularizations, by showing the number of GMRES iterations performed.

In each table below,

(a) is the total number of GMRES solvers used when the number of the outer-loop iteration of the modified Uzawa algorithm is $n=5$; 
TABLE 4.1

Averaged number of iterations in each GMRES solver using $H^{1}$-seminorm regularization.

\begin{tabular}{|c||c|c||c|c|}
\hline \multicolumn{1}{|c||}{} & \multicolumn{2}{c||}{ Without preconditioning } & \multicolumn{2}{c|}{ With preconditioning } \\
\cline { 2 - 5 }$h$ & (a) & (b) & (a) & (b) \\
\hline \hline $1 / 80$ & 65 & 132 & 66 & 132 \\
\hline $1 / 160$ & 65 & 215 & 67 & 160 \\
\hline $1 / 320$ & 65 & 377 & 67 & 166 \\
\hline
\end{tabular}

TABLE 4.2

Averaged number of iterations in each GMRES solver using $H^{1}$-full-norm regularization.

\begin{tabular}{|c||c|c||c|c|}
\hline \multicolumn{1}{|c||}{} & \multicolumn{2}{c||}{ Without preconditioning } & \multicolumn{2}{c|}{ With preconditioning } \\
\cline { 2 - 5 } & (a) & (b) & (a) & (b) \\
\hline \hline $1 / 80$ & 66 & 132 & 67 & 122 \\
\hline $1 / 160$ & 66 & 215 & 66 & 146 \\
\hline $1 / 320$ & 69 & 376 & 67 & 156 \\
\hline
\end{tabular}

TABLE 4.3

Averaged number of iterations in each GMRES solver using $H^{1}$-seminorm regularization.

\begin{tabular}{|c||c|c||c|c|}
\hline \multicolumn{1}{|c||}{} & \multicolumn{2}{c||}{ Without preconditioning } & \multicolumn{2}{c|}{ With preconditioning } \\
\cline { 2 - 5 } & (a) & (b) & (a) & (b) \\
\hline \hline $1 / 80$ & 57 & 123 & 57 & 115 \\
\hline $1 / 160$ & 57 & 203 & 57 & 129 \\
\hline $1 / 320$ & 57 & 363 & 57 & 135 \\
\hline
\end{tabular}

(b) represents the average number of iterations within one GMRES solver, i.e., the total number of iterations accumulated in all GMRES iterations divided by the number given in (a).

Example 8. The observed data $z$ and the exact coefficient $q(x)$ are taken to be the same as in Example 1. Results by using $H^{1}$-seminorm and full-norm regularizations with or without preconditioning are shown in Tables 4.1 and 4.2 .

For all the examples given in this section, the graphs of the numerically identified solution $q_{h}(x)$ are similar to the ones we have shown in section 4.1, so we do not present them here again. From Tables 4.1-4.2, we see that the numbers of GMRES solvers performed (column (a)) are approximately the same with different mesh sizes, which means the convergence of the algorithm is almost independent of finite element mesh sizes; this seems to be a surprising observation. When considering the average number of iterations within one GMRES solver (column (b)), the one with preconditioning seems to have much fewer iterations, and this averaged number of iterations seems to be asymptotically constant. This indicates the preconditioners we used are almost optimal, namely, the numbers of the iterations of the preconditioned GMRES required to reach the stopping criteria are independent of the finite element mesh sizes. So the advantage of the preconditioning is more obvious when the problem sizes are larger. We can see such conclusion more evidently when we compare the results in Tables 4.1-4.2 and Tables 4.3-4.4 for the one-dimensional problems with the results in Tables 4.5-4.6 and Tables 4.7-4.8 for the two-dimensional problems.

The above observations are true for all other examples shown below.

Example 9. We take the observed data $z$ and the true parameter $q(x)$ (discontinuous) to be the same as in Example 2. Results using $H^{1}$-seminorm and full-norm regularizations with or without preconditioning are shown in Tables 4.3 and 4.4.

Example 10. We take the observed data $z$ and the true coefficient $q(x, y)$ to be 
TABLE 4.4

Averaged number of iterations in each GMRES solver using $H^{1}$-full-norm regularization.

\begin{tabular}{|c||c|c||c|c|}
\hline \multicolumn{1}{|c||}{} & \multicolumn{2}{c||}{ Without preconditioning } & \multicolumn{2}{c|}{ With preconditioning } \\
\cline { 2 - 5 } & (a) & (b) & (a) & (b) \\
\hline \hline $1 / 80$ & 57 & 123 & 57 & 112 \\
\hline $1 / 160$ & 57 & 204 & 57 & 127 \\
\hline $1 / 320$ & 57 & 363 & 57 & 131 \\
\hline
\end{tabular}

TABLE 4.5

Averaged number of iterations in each GMRES solver using $H^{1}$-seminorm regularization.

\begin{tabular}{|c||c|c||c|c|}
\hline \multicolumn{1}{|c||}{$h$} & \multicolumn{2}{c||}{ Without preconditioning } & \multicolumn{2}{c|}{ With preconditioning } \\
\cline { 2 - 5 } & (a) & (b) & (a) & (b) \\
\hline \hline $1 / 10$ & 42 & 143 & 41 & 125 \\
\hline $1 / 20$ & 45 & 446 & 45 & 227 \\
\hline $1 / 30$ & 45 & 830 & 45 & 269 \\
\hline $1 / 40$ & 45 & 1122 & 44 & 276 \\
\hline
\end{tabular}

TABLE 4.6

Averaged number of iterations in each GMRES solver using $H^{1}$-full-norm regularization.

\begin{tabular}{|c||c|c||c|c|}
\hline \multicolumn{1}{|c||}{$h$} & \multicolumn{2}{c||}{ Without preconditioning } & \multicolumn{2}{c|}{ With preconditioning } \\
\cline { 2 - 5 } & (a) & (b) & (a) & (b) \\
\hline \hline $1 / 10$ & 41 & 143 & 41 & 110 \\
\hline $1 / 20$ & 45 & 446 & 45 & 207 \\
\hline $1 / 30$ & 45 & 830 & 44 & 236 \\
\hline $1 / 40$ & 45 & 1122 & 44 & 247 \\
\hline
\end{tabular}

TABLE 4.7

Averaged number of iterations in each GMRES solver using $H^{1}$-seminorm regularization.

\begin{tabular}{|c||c|c||c|c|}
\hline \multicolumn{1}{|c||}{$h$} & \multicolumn{2}{c||}{ Without preconditioning } & \multicolumn{2}{c|}{ With preconditioning } \\
\cline { 2 - 5 } & (a) & (b) & (a) & (b) \\
\hline \hline $1 / 10$ & 42 & 145 & 42 & 130 \\
\hline $1 / 20$ & 49 & 449 & 49 & 245 \\
\hline $1 / 30$ & 50 & 840 & 50 & 284 \\
\hline $1 / 40$ & 50 & 1149 & 50 & 295 \\
\hline
\end{tabular}

TABLE 4.8

Averaged number of iterations in each GMRES solver using $H^{1}$-full-norm regularization.

\begin{tabular}{|c||c|c||c|c|}
\hline \multicolumn{1}{|c||}{$h$} & \multicolumn{2}{c||}{ Without preconditioning } & \multicolumn{2}{c|}{ With preconditioning } \\
\cline { 2 - 5 } & (a) & (b) & (a) & (b) \\
\hline \hline $1 / 10$ & 43 & 144 & 42 & 114 \\
\hline $1 / 20$ & 49 & 449 & 49 & 224 \\
\hline $1 / 30$ & 50 & 840 & 50 & 266 \\
\hline $1 / 40$ & 50 & 1148 & 50 & 282 \\
\hline
\end{tabular}

the same as in Example 3. Results using $H^{1}$-seminorm and full-norm regularizations with or without preconditioning are shown in Tables 4.5 and 4.6.

Example 11. The observed data $z$ and the true parameter $q(x, y)$ (discontinuous) are taken to be the same as in Example 4. Results using $H^{1}$-seminorm and full-norm regularizations with or without preconditioning are shown in Tables 4.7-4.8.

Acknowledgments. The authors wish to thank an anonymous referee and the editor for many constructive comments that improved this paper. 


\section{REFERENCES}

[1] T. F. Chan and T. P. Mathew, Domain decomposition algorithms, Acta Numer., Cambridge University Press, Cambridge, UK, 1994, pp. 61-143.

[2] T. F. Chan And P. Mulet, On the convergence of the lagged diffusivity fixed point method in total variation image restoration, SIAM J. Numer. Anal., 36 (1999), pp. 354-367.

[3] T. F. Chan And X.-C. TAI, Augmented Lagrangian and Total Variational Methods for Recovering Discontinuous Coefficients from Elliptic Equations, Technical Report CAM 97-2, Dept. of Math., University of California at Los Angeles, 1997.

[4] T. F. Chan And X.-C. TAI, Identification of Discontinuous Coefficients from Elliptic Problems Using Total Variation Regularization, Technical Report CAM 97-35, Dept. of Math., University of California at Los Angeles, 1997.

[5] Z. Chen AND J. Zou, An augmented Lagrangian method for identifying discontinuous parameters in elliptic systems, SIAM J. Control Optim., 37 (1999), pp. 892-910.

[6] Z. Chen AND J. Zou, Finite element methods and their convergence for elliptic and parabolic interface problems, Numer. Math., 79 (1998), pp. 175-202.

[7] P. G. Ciarlet, Basic error estimates for elliptic problems, in Handbook of Numerical Analysis, P. G. Ciarlet and J.-L. Lions, eds., Vol. II, North Holland, Amsterdam, 1991, pp. 17-352.

[8] D. C. Dobson And C. R. Vogel, Convergence of an iterative method for total variation denoising, SIAM J. Numer. Anal., 34 (1997), pp. 1779-1791.

[9] S. C. Eisenstat, H. C. Elman, and M. H. Schultz, Variational iterative methods for nonsymmetric systems of linear equations, SIAM J. Numer. Anal., 20 (1983), pp. 345-357.

[10] K.-H. Hoffmann And J. Zou, Parallel solution of variational inequality problems with nonlinear source terms, IMA. J. Numer. Anal., 16 (1996), pp. 31-45.

[11] K. Itô, M. KRoller, And K. Kunisch, A numerical study of an augmented Lagrangian method for the estimation of parameters in elliptic systems, SIAM J. Sci. Comput., 12 (1991), pp. 884-910.

[12] K. ITÔ AND K. KUNISCH, The augmented Lagrangian method for parameter estimation in elliptic systems, SIAM J. Control Optim., 28 (1990), pp. 113-136.

[13] K. Itô And K. Kunisch, Augmented Lagrangian-SQP-methods in Hilbert spaces and application to control in the coefficients problems, SIAM J. Optim., 6 (1996), pp. 96-125.

[14] Y. Keung And J. Zou, Numerical identifications of parameters in parabolic systems, Inverse Problems, 14 (1998), pp. 83-100.

[15] K. Kunisch AND X.-C. TAI, Sequential and parallel splitting methods for bilinear control problems in Hilbert spaces, SIAM J. Numer. Anal., 34 (1997), pp. 91-118.

[16] Y. SAAD, Iterative Methods for Sparse Linear Systems, PWS Publishing Company, Boston, 1996.

[17] Y. SAAD AND M. H. SChultz, GMRES: A generalized minimum residual algorithm for solving nonsymmetric linear systems, SIAM J. Sci. Comput., 7 (1986), pp. 856-869.

[18] X.-C. TAi, J. Frøyen, M. Espedal, and T. Chan, Overlapping domain decomposition and multigrid methods for inverse problems, Contemp. Math, 218 (1998), pp. 523-529. 